\title{
Detection of $\beta$-lactamases producing Methicillin-Resistant Staphylococcus aureus (MRSA)
}

\author{
and Mee'aad K. Al-Kudheiri ${ }^{3 *}$ Ali M. Al-Mohana ${ }^{1}$, Azhar N. Hussein ${ }^{2}$ \\ ${ }^{1}$ Department of Microbiology, College of Medicine, Kufa University, Iraq. \\ ${ }^{2}$ Department of Microbiology, College of Education, Qadisiya University, Iraq. \\ ${ }^{3}$ Department of Community Health, Kufa Technical Institute, Iraq.
}

\section{Received:4/12/2014}

Accepted:-2/2/2015

\begin{abstract}
Methicillin resistant Staphylococcus aureus (MRSA) is one of the most important causes of nosocomial and community infections and most clinical isolates are $\beta$-lactams and multidrug resistant. Resistance to $\beta$-lactam antibiotics is most often due to penicillin binding proteins (PBPs) and $\beta$-lactamases production. Characterization of $\beta$-lactamases is important for choosing appropriate antibiotic therapy, therefore $135 \mathrm{~S}$. aureus were collected from 343 clinical samples between August 2012 and January 2013 from several clinical sources that were randomly selected from patients in three main hospitals in AlNajaf city and the prevalence of MRSA in Najaf hospitals was $64(47.4 \%)$ and the remaining $71(52.6 \%)$ isolates were MSSA, and all MRSA isolates were tested for $\beta$ lactamase production by using seven methods: [cloverleaf test, Masuda double-disc test, iodometric method, acidometric method, chromogenic (nitrocefine) method, double disc method and penicillin disc diffusion method]. The findings of this study revealed that chromogenic (nitrocefine) method, cloverleaf test and Masuda test had a high accuracy $(100 \%)$ comparative with other methods tested for detection of MRSA $\beta$-lactamase production.
\end{abstract}

\section{INTRODUCTION}

Both MRSA and methicillin sensitive $S$. aureus (MSSA) can produce $\beta$ lactamases. These Enzymes alone may be responsible for borderline methicillin and oxacillin resistance phenotype even in strains without PBP2. Most MRSA strains produce $\beta$-lactamase in addition to PBP2 (1). Staphylococci are most commonly produce $\beta$-lactamase, one of the major mechanisms of resistance to $\beta$ lactam antibiotics. Approximately most of clinical isolates are resistant to $\beta$ lactams as a result of enzyme production.
The enzyme is excreted into the surrounding environment where the hydrolysis of $\beta$-lactams takes place before the drug can bind to PBPs in the cell membrane (2). The transcription of $m e c A$ and blaZ resistance genes may be controlled by homologous twocomponent systems consisting on a sensor-inducer BlaR1 and MecR1 and a repressor BlaI and MecI. Interestingly, in spite of the cross-resistance to virtually all $\beta$-lactams provided by $m e c A$, the great majority more than $(95 \%)$ of contemporary MRSA are still positive for the $\beta$-lactamase locus ${ }^{3}$. Moreover, 
the regulators of blaZ, BlaR1 and BlaI, can efficiently induce mecA transcription and, do it faster than the natural $m e c A$ regulators, MecR1 and MecI. In addition, since many MRSA strains do not have functional mecI$m e c R l$ genes due to polymorphisms in the $m e c A$ regulatory region, the mecA transcription is presumably under the control of the blaI-blaRl genes only. The presence of the blaZ locus has been shown to promote mecA acquisition and stabilization ${ }^{(3.4)}$. Several methods are available to detect $\beta$-lactamase production in bacteria. The present study was aimed to evaluate seven phenotypic methods for the detection of $\beta$-lactamase producing MRSA was isolated from Najaf hospitals.

\section{MATERIALS AND METHODS}

\section{Bacterial isolates}

A total of $135 S$. aureus isolates were isolated between August 2012 and January 2013 from several clinical sources including the urine, skin, burn, wounds, sputum, diabetic foot ulcer, nares and vagina that were randomly selected from patients in Al-Zaharaa, AlHakeem and Al-Sader hospitals in AlNajaf city. Isolates were identified depending on the morphological features on culture medium and biochemical tests according to the classification of Bergey's manual (5) and MacFaddin (5).

\section{$\beta$-Lactamase Production Tests}

\section{Cloverleaf Test}

Muller-Hinton agar [(MHA), Himedia, India] plate was inoculated with [Escherichia coli (E. coli) American type culture collection (ATCC) 25922]. A penicillin disc [(10U), Himedia, India] was placed in the center of the plate and four test isolates were streaked radially outward from the disc to produce growth about $0.25 \mathrm{~cm}$ wide. The plate incubated at $37^{\circ} \mathrm{C}$ for 18 hours and examined, if the isolate produced $\beta$-lactamase, giving rise to a cloverleaf pattern ().

\section{Masuda Double-Disc Test}

In this test E. coli ATCC 25922 was swabbed on the plate and a penicillin disc $(10 \mathrm{U})$ placed in the center. Filter paper discs containing test isolates were placed $10 \mathrm{~mm}$ away from the central disc. Distortion of inhibition around the centrally placed disc by the discs containing test indicates positive $\beta$ lactamase production ${ }^{(8)}$.

\section{Iodometric Method}

This method was done by using iodometric kit (IVD, Syria), $100 \mu 1$ of the penicillin solution was dispensed into a well of a microtitre plate. Several colonies of the organism to be tested were emulsified into the solution to get dense suspension. Two drops of starch were added and then the plate was kept at room temperature for 30 minutes. One drop of iodine was added, which turn the solution blue. If the blue color disappeared in 10 minutes, the organism was considered as $\beta$-lactamase positive. Negative control with penicillin alone was kept without any culture suspension (7).

\section{Acidometric Method}

Solution was prepared by adding $2 \mathrm{ml}$ of the $(0.5 \%)$ phenol red solution $(\mathrm{BDH}$, England) to $16.6 \mathrm{ml}$ sterile D.W. After mixing, this solution was added to a vial of penicillin $\mathrm{G}$ (Ras Al Khaimah, U.A.E.), which contained 20 million units. Since this solution was at an acidic $\mathrm{pH}$ due to citrate buffer in the penicillin, $1 \mathrm{~N}$ of $\mathrm{NaOH}$ (Fluka, Switzerland) was added drop-wise to the solution still development of reddish violet color $\mathrm{pH}$ 8.5. It was used for the detection of bacterial ability to produce $\beta$-lactamase enzyme. Penicillin phenol red solution 
$(100 \mu \mathrm{l})$ was placed in the well of microtitre plate. Several colonies were suspended in the solution to get dense suspension. The solution turned yellow within 15 minutes if $\beta$-lactamase enzyme was produced (?).

\section{Chromogenic (Nitrocefin) Disc Method}

This test was done by using sterile forceps to place a nitrocefin disc (Fluka, Switzerland) on a clean slide; a drop of D.W was added to the disc and streaked induced growth with nitrocefin disc. Induced growth was taken from the zone margin surrounding an oxacillin or cefoxitin disc test on MHA plate after 18 hours of incubation. $\beta$-lactamase production was noticed by the appearance a pink color with 15 minutes (9.10).

\section{Double-Disc Synergy Test (DDST)}

The production of $\beta$-lactamase enzyme was detected by performing DDST that employs a combination of piperacillin (PI) $\quad 100 / 10 \quad \mu g \quad$ and piperacillin/tazobactam (PI/PIT) 100/10 $\mu \mathrm{g}$ discs (Himedia, India). A 0.5 McFarland bacterial suspension (Fluka, Switzerland) was prepared with normal saline for each isolate and inoculated on MHA plate. PI and PI/PIT discs were placed $15 \mathrm{~mm}$ apart edge to edge manually. Plates were read after 18 hours of incubation at $35^{\circ} \mathrm{C}$. $\quad \beta$-lactamase production was noticed by the appearance a synergism reaction ${ }^{(11)}$.

\section{Penicillin Disc Diffusion Test}

It is based on the method originally described by Bauer et al. (1966). This is the most thoroughly described D.D method on MHA medium for which interpretive standards have been developed and supported by CLSI data. MHA plate was inoculated with isolate under study. A penicillin disc (10U) was placed on the surface of the plate. The plate incubated at $37^{\circ} \mathrm{C}$ for 18 hours and examined, if the isolate produced $\beta$ lactamase, giving sharp edge around the disc to ${ }^{(10)}$.

\section{RESULTS AND DISCUSSION}

Several methods are available to detect $\beta$-lactamase production in bacteria. The present study was conducted in order to evaluate seven phenotypic methods for the detection of $\beta$-lactamase produced by 64 MRSA isolated from Najaf hospitals. Results revealed that all MRSA were resistant to penicillin. However, $100 \%$ were $\beta$ lactamase positive by nitrocefin discs, cloverleaf, and Masuda double-disc, $90.6 \%$ by iodometric, $84.4 \%$ by acidimetric, $45.3 \%$ by double-disc synergy, and only $4.7 \%$ by penicillin disc zone edge (Table 1).

Chromogenic method is one of the successful method based on the principle that hydrolysis of certain $\beta$ lactam antibiotic leads to a distinct color change from a yellow to a deep red color (Figure 1). Nitrocefin disc utilized in the chromogenic method has been reported to be effective in detecting all known $\beta$ lactamase ${ }^{(10,12)}$.

Table 1. Comparative test results of the seven phenotypic methods to detect $\beta$-lactamase production in 64 MRSA isolates 


\begin{tabular}{|l|l|}
\hline Phenotypic test & Positive No.(\%) \\
\hline Nitrocefin disc & $64(100)$ \\
\hline \multicolumn{1}{|c|}{ Cloverlea } & $64(100)$ \\
\hline Masuda double-disc & $64(100)$ \\
\hline odometric & $58(90.6)$ \\
\hline Acidimetric & $54(84.4)$ \\
\hline enicillin disc zone edge & $3(4.7)$ \\
\hline pouble-disc synergy (PI/ PIT) & $29(45.3)$ \\
\hline
\end{tabular}

The rate of nitrocefin tests in the current study was $100 \%$, this is similar to findings reported by Odonkor and Addo (9) In contrast, other studies reported lower rates results as compared with current investigation, $85.7 \%{ }^{(13)}$, $88 \%$ (14) and $75 \%{ }^{(15)}$. The rates may be differing from study to other, depending on the manufacturer of the nitrocefin assay ${ }^{(1)}$. It is likely that the factors affecting nitrocefin detection method are less of a problem. Furthermore, its high sensitivity, specificity, easy-to-read and rapid result with lack of need for preparation of the reagent makes it a good choice for $\beta$-lactamase activity detection ${ }^{(12)}$.

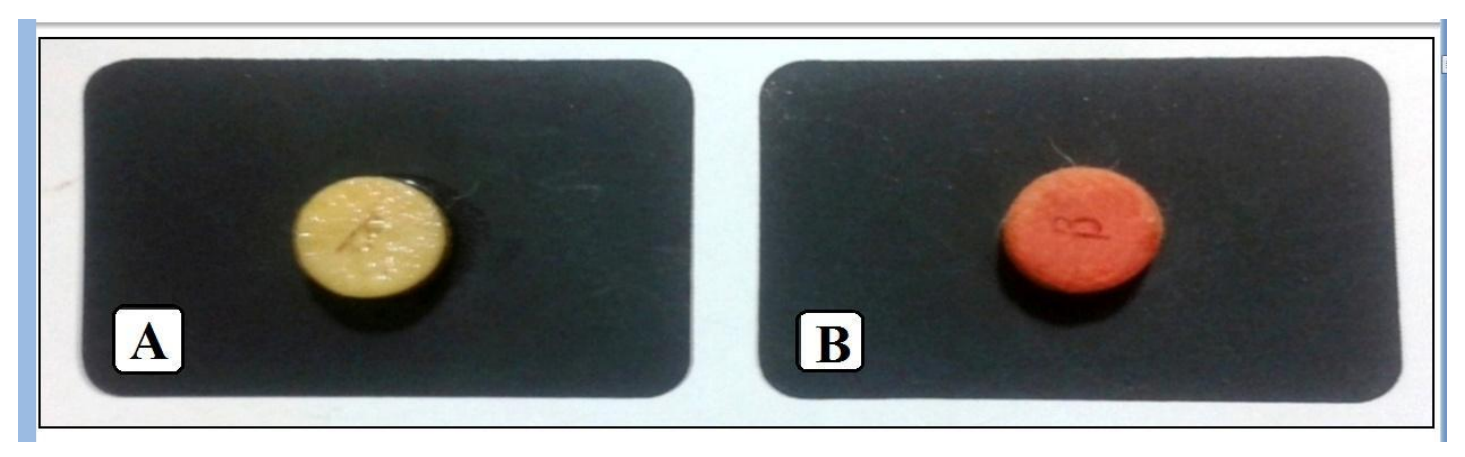

Figure 1. $\beta$-lactamases production detected by nitrocefin disc method.

(A) Negative result, no change in color of nitrocefin disc and still keeping the yellow color by ATCC 25922 as negative control; (B) Positive result, color of nitrocefin disc changed from yellow to pink within 15 minutes by isolate (NJ-34).

In cloverleaf method, if the test isolate produced $\beta$-lactamase, the growth coincide thus giving rise to a cloverleaf pattern (Figure 2). In Masuda method positive result for $\beta$-lactamase production appeared distortion of inhibition around the centrally placed disc (Figure 3). These two methods also had high rates similar to the chromogenic method. In concordance to this finding, a 
high sensitivity and specificity (100\%) for these methods has been reported recently (10, 17). In the current study, cloverleaf technique did not get any false results and this agreed with the fact that this test depend on the $\beta$-lactamase produced by one organism allowing an indicator isolate to grow, there is nil chance of getting false positive results, additionally, this technique found to be the easy, cost effective, and reliable method for detection of $\beta$ lactamase. This result was confirmed with Samant and PAI ${ }^{(7)}$.

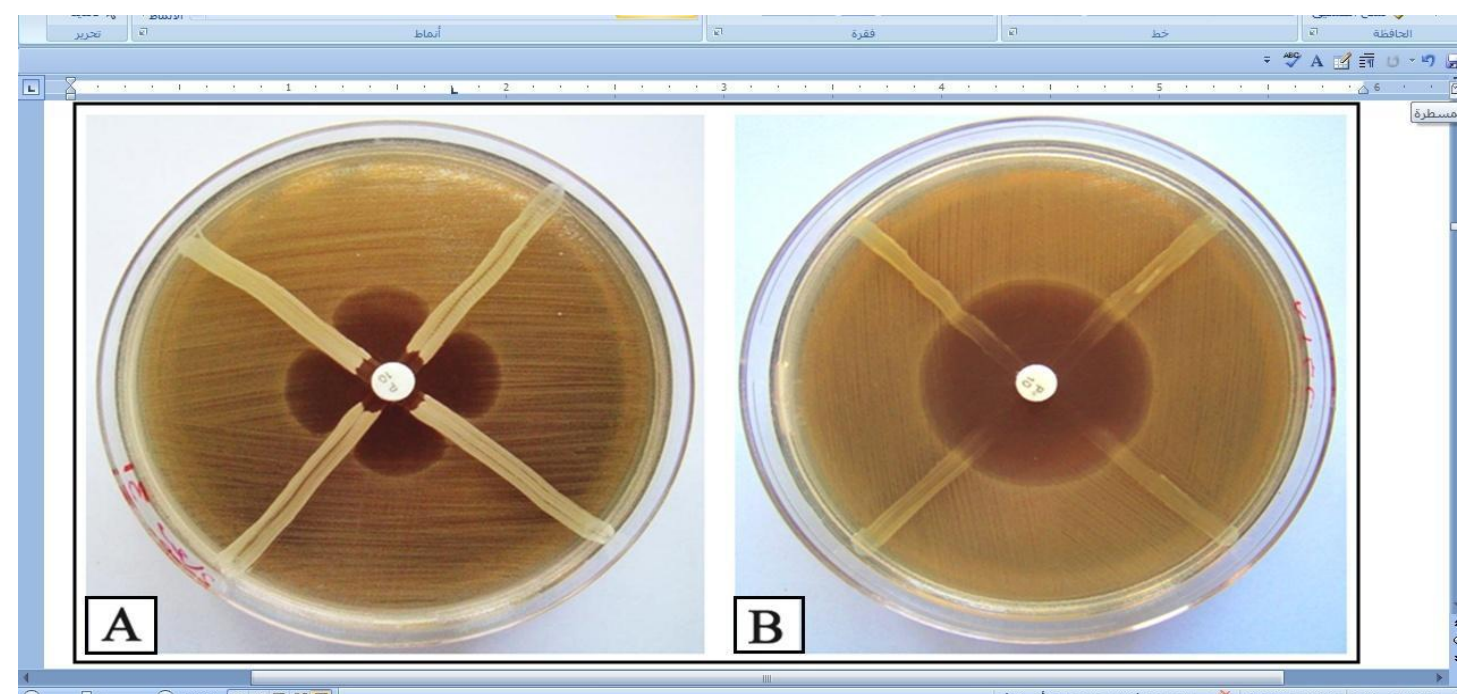

(7) E

Figure 2. $\beta$-lactamases production detected by cloverleaf method. (

A) Positive result, giving rise to a cloverleaf pattern around [penicillin (P10U)] disc by isolates NA-47, 95, 96 and 99); (B) Negative result, no cloverleaf pattern produced by ATCC 25922 as negative control.

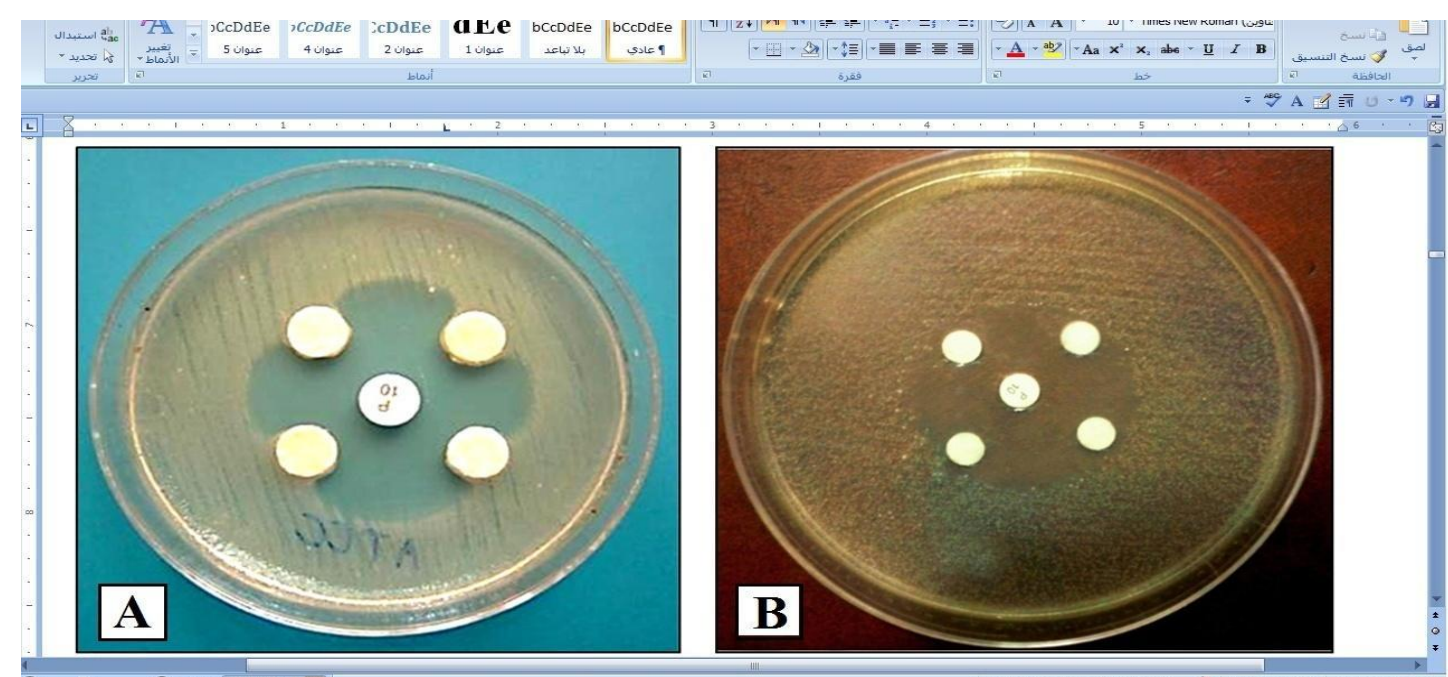

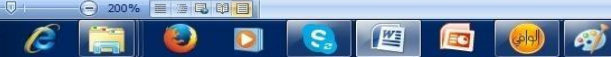

Figure

3. $\quad \beta$-lactamases

production

detected

by Masuda

method. 
A) Isolates (NJ-47, 95, 96 and 99) showed positive result for $\beta$-lactamase production giving rise to a distortion of inhibition around the centrally [penicillin (P 10U)] disc; (B) Absence of an irregular inhibition zone was considered as a positive result, ATCC 25922 was used as negative control.

Acidimetric is one of common method uses a $\mathrm{pH}$ indicator color change from purple pink to yellow to detect the formation of at least one extra carboxyl group produced during the hydrolysis of $\beta$-lactam antibiotic by $\beta$-lactamase, also the iodometric method detects the loss of blue color from a blue starch/iodine complex caused by the removal of iodine from the complex by the reducing action of a $\beta$-lactamase hydrolysis product (Figure $4 \mathrm{~A}$ and $\mathrm{B}$ ).
Of note, the rates of acidimetric and iodometric obtained in the present study are slightly lower than the above three tests. In iodometric and acidimetric tests, penicillin is used as the substrate and, therefore, the tests are equipped to detect penicillinases. The higher rate of chromogenic method compared to these two tests could be due to the detection of both penicillinase and cephalosporinase enzymes produced by the test isolates in the chromogenic test ${ }^{(10)}$, (Figure 5). The present finding of better rate of the iodometric method compared to acidimetric is supported by report that iodometric tests are more accurate and sensitive than acidimetric methods ${ }^{(18)}$.

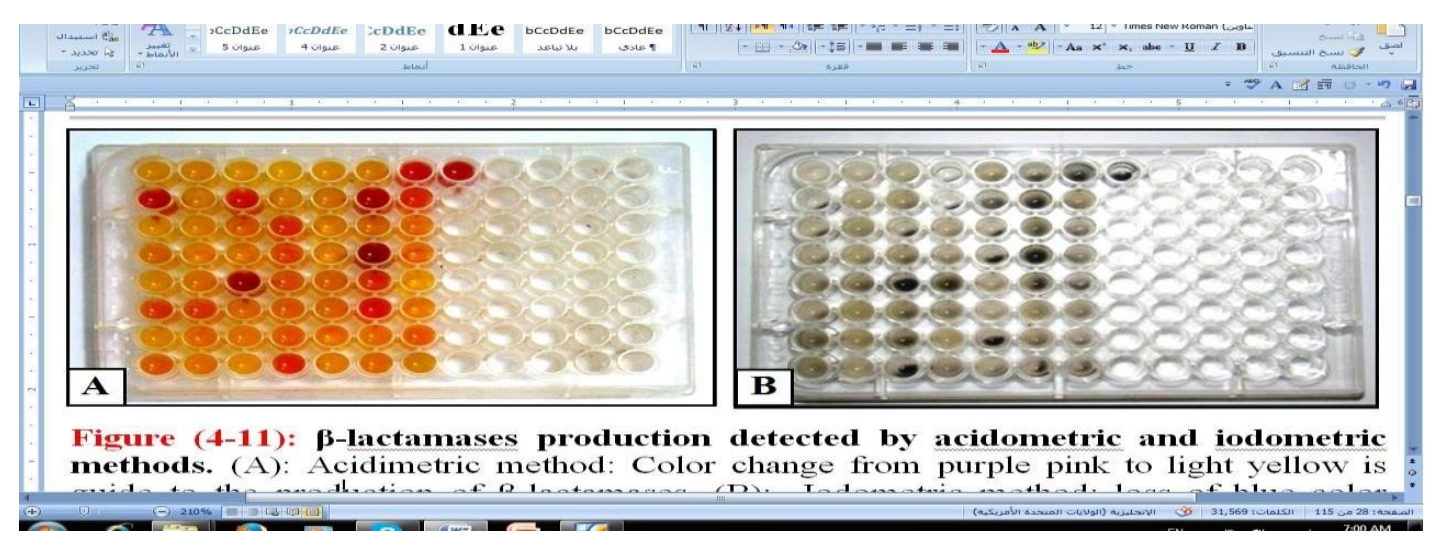

Figure 4. Results of $\beta$-lactamases production by acidimetric and iodometric methods.

(A) Acidimetric method, positive S. aureus isolates represented by change color from purple pink to light yellow; (B) Iodometric method, positive $S$. aureus isolats represented by loss of blue color from starch-iodine complex.

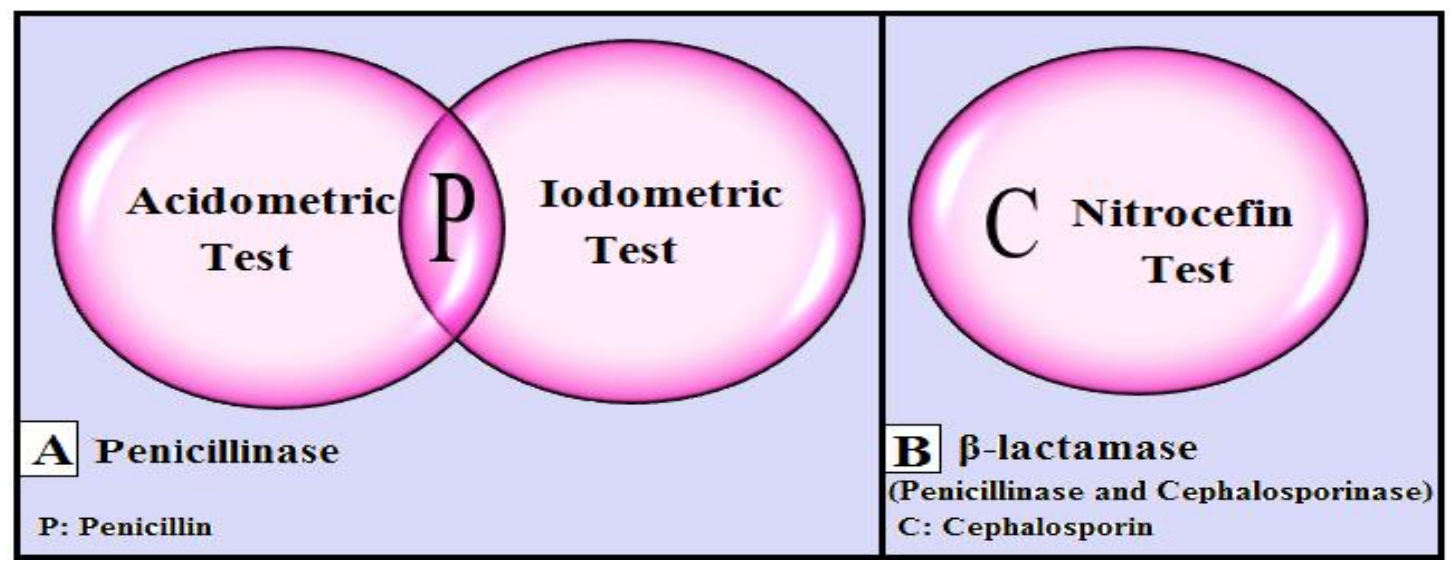

Figure 5. $\beta$-lactamases detection by conventional phenotypic methods. 
A) Acidometric and Iodometric tests are used penicillin as the substrate therefore, the tests are detect penicillinases; (B) Nitrocefin test is used cephalosporin as the substrate could be due to the detection of both penicillinase and cephalosporinase enzymes.

Most laboratories perform penicillin susceptibility testing of staphylococcal isolates by agar disc diffusion. Various investigators have suggested that $\beta$ lactamase production can be predicted by the appearance of the inhibitory zone edge around a penicillin or ampicillin disc ${ }^{(10,19)}$. CLSI reported that test must be reporting as penicillin resistance if penicillin inhibition zones are $\geq 29 \mathrm{~mm}$ or less and zone edge showing sharply demarcated zone edge with fully developed individual colonies within the inhibition zone ${ }^{(1)}$. Zone edges with this appearance suggest that the organism tested is a $\beta$-lactamase producer. Table (1) and Figure (6) show results of the penicillin disc diffusion zone edge test carried out on all the 64 MRSA isolates. Out of the isolates, $3(4.7 \%)$ produced $\beta$ lactamase. In MRSA the penicillin zone edge test considered unhelpful for detecting $\beta$-lactamase production because MRSA be resistant to penicillins and cephalosporins and inhibition zone around penicillin disc either absence or very small, for this reason, the prediction is difficult and sometimes impossible. Therefore, the rate of the penicillin zone edge test in the current study did not exceed $4.7 \%$. The main reason for the use of this test in the current study for compare between common conventional phenotypic tests to find the best method that can predict the isolate producing $\beta$ lactamase.

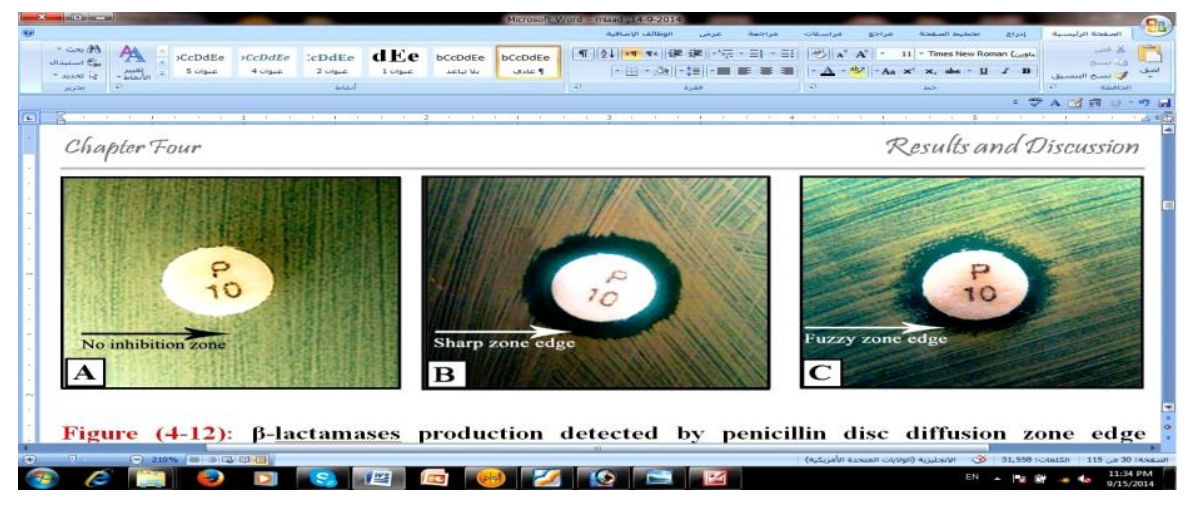

Figure 6. $\beta$-lactamases production detected by penicillin disc diffusion zone edge method. (

A) No inhibition zone was seen around [penicillin ( $\mathrm{P} 10 \mathrm{U})$ ] disc that represented constitutive resistance by 61 isolates in the present study; (B) Sharply demarcated zone edge with fully developed individual colonies within the inhibition zone. Zone edges with this appearance suggest that the isolate $\mathrm{NJ}-20$ a tested is a $\beta$-lactamase producer; (C) Zone edge showing gradual tapering of growth. Zone edges with this appearance suggest that the MSSA isolate $\mathrm{NJ}-8 \mathrm{a}$ tested is not a $\beta$-lactamase producer.
Production of staphylococcal $\beta$ lactamase is generally inducible, which means that colonies must grow in the presence of a $\beta$-lactam antibiotic. The inducible resistance was encouraged many researchers to find easy, fast and low-cost and applicable tests. The only study was introduced by Sharma and Mall (11) performing double-disc synergy test that employs a combination of

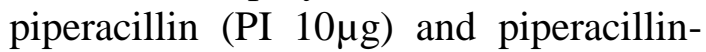


tazobactam (PIT 100/10 $\mu$ g) for detection the production of $\beta$-lactamase in MRSA. The present study indicates that the rate of MRSA isolates producing $\beta$-lactamase enzyme was $45.3 \%$, which is low (Table 1 and Figure 7). This may be the MRSA isolates are resistant to all kinds of $\beta$ lactams antibiotics, but in some cases showed inhibition zones around piperacillin and piperacillin-tazobactam
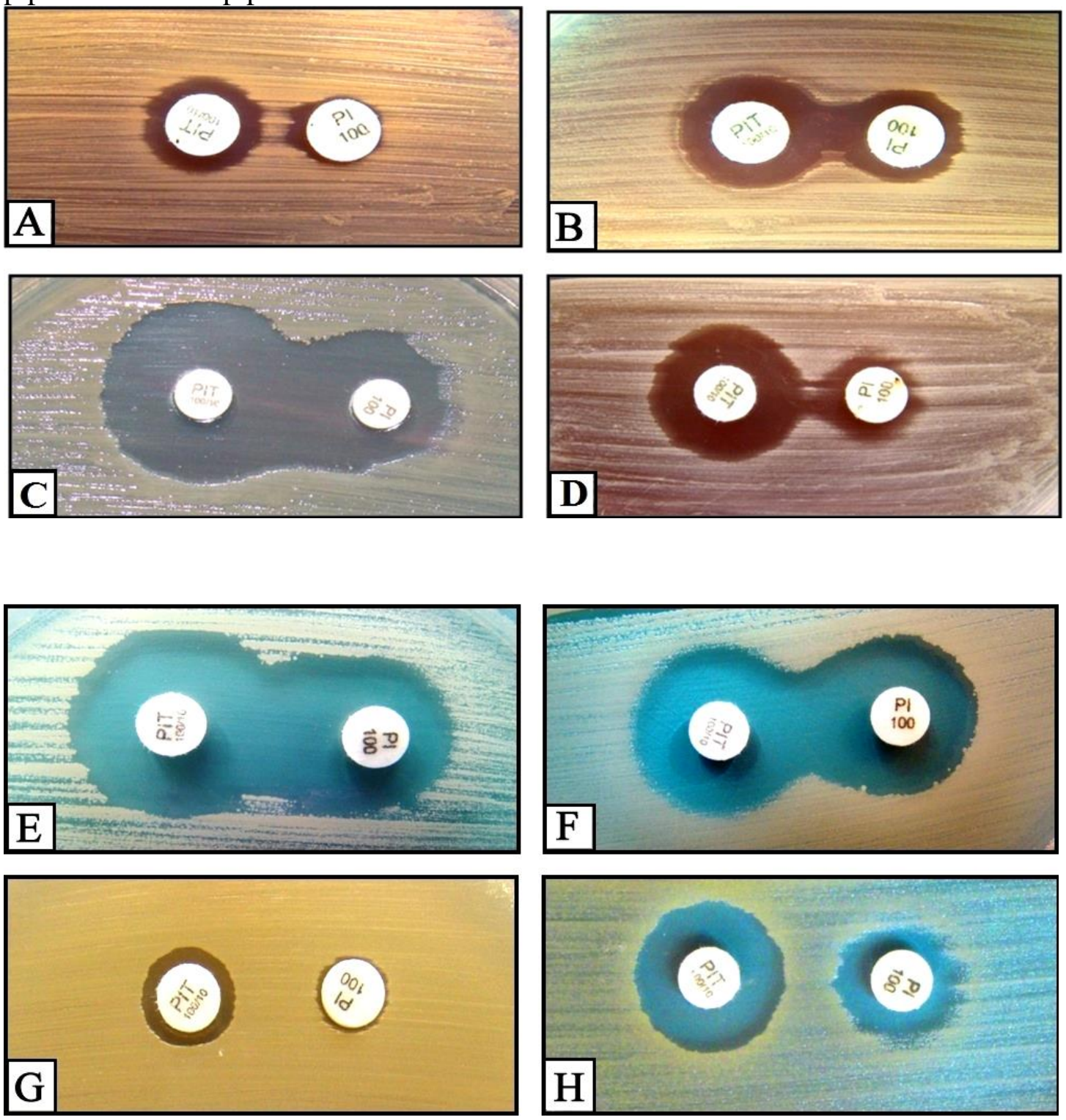

Figure 7. $\beta$-lactamases detection by double-disc synergy test that employs a combination of

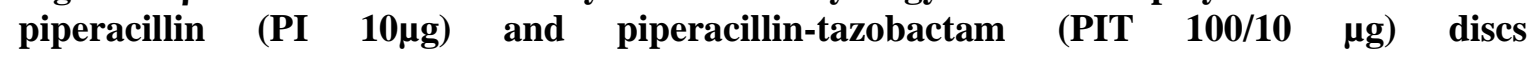

- A, B, C, D, E, and F give positive results for $\beta$-lactamase production in MRSA isolates $\mathrm{NJ}-65,70,57,78,3$ and 59 respectively; (G) Constitutive resistance to invitro, that's not mean the MRSA are sensitive for this antibiotics because MRSA could be resistant to all antibiotics invivo and may be sensitive invitro, this fact was confirmed by (10). Therefore, the prediction of $\beta$-lactamase production in MRSA isolates is difficult, not easy, inaccurate, and impractical in the most time.
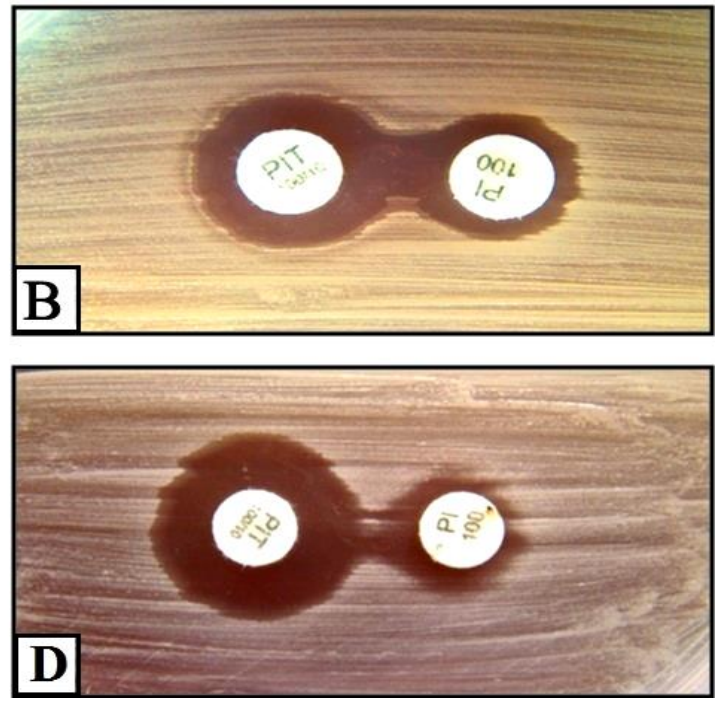

D

PI and PIT in isolate NJ-99; (H) Negative result in ATCC 25922 isolates was used as negative control. All these results were confirmed based on CLSI guideline ${ }^{(10)}$. 


\section{Conclusion}

In brief, with high rates, the study indicates that the nitrocefin discs, cloverleaf, and Masuda double-disc were accurate and superior methods in the detection of $\beta$-lactamase producing $S$. aureus and suggest that these methods done as routine tests in Najaf laboratories.

\section{REFERENCES}

1- Borhani, K., Eftekhar, F. and Monfared, S.D. (2009). $\beta$-lactamase typing by substrate hydrolysis in clinical isolates of methicillin resistant Staphylococcus aureus. Iranian. J. Clinic. Infect. Dis. 4(4): 202206.

2- Deurenberg, R. H., Vink, C., Kalenic, S., Friedrich, A. W., Bruggeman, C. A. and Stobberingh, E. E. (2007). The molecular evolution of methicillin resistant Staphylococcus aureus. Clinic. Microbiol. Infect. 13: 222-235.

3- International working group on the classification of staphylococcal cassette chromosome elements (IWG-SCC) (2009). Classification of staphylococcal cassette chromosome mec: guidelines for reporting novel SCCmec elements. Antimicrob. Agents. Chemother. 53(12): 4961-4967.

4- Milheiriço, C., Oliveira, D.C. and de Lencasatre H. (2007). Update to the multiplex polymerase chain reaction (PCR) strategy for assignment of mec element types in Staphylococcus aureus. Antimicrob. Agents. Chemother. 51(9): 3374-3377.

5- Holt, J.G., Krieg, N.R., Sneath, P.H., Staley, J.T. and Williams, S.T. (1994). Bergey's manual of determinative bacteriology, 9th edn. Baltimore, MD: Williams and Williams.
6- MacFaddin, J. F. (2000). Biochemical tests for identification of medical bacteria 3rd ed. Lippincott Williams and Wilkins, USA.

7- Samant, S.A. and PAI, C.G. (2012). Comparative evaluation of $\beta$-lactamase 238 ection methods in Staphylococci. Int. J. Kes. Pharm. Biomed. Sciences. 3(4):15801588.

8- Livermorea, D.M. and Brown, D.F.J. (2001). Detection of $\beta$-lactamase-mediated resistance. J. Antimicrob. Chemother. 48(1): 59-64.

9- Odonkor, S.T. and Addo, K.K. (2011). Evaluation of three methods for detection of methicillin resistant Staphylococcus aureus. Int. J. Biol. Med. Res. 2(4): 1031-1034.

10- Clinical and laboratory standards institute. (CLSI) (2012). Performance standards for antimicrobial susceptibility testing. M100S22. Clinical and laboratory standards institute, Wayne, PA.

11- Sharma, S. and Mall, A. (2011). The prevalence, antibiogram and characterization of methicillin resistant Staphylococcus aureus among the patients from the Doon Valley hospitals. African. J. Microbiol. Res. 5(21): 3446-3451.

12- Bidya, S. and Suman, R.S. (2014). Comparative study of three $\beta$-Lactamase test methods in Staphylococcus aureus isolated from two Nepalese hospitals. Open. J. Clinic. Diagnos. 4(1): 47-52.

13- Broekema, N.M., Van, T.T., Monson, T.A., Marshall, S.A. and Warshauer, D.M. (2009). Comparison of cefoxitin and oxacillin disk diffusion methods for detection of mecA mediated resistance in Staphylococcus aureus in a large-scale study. J. Clin. Microbiol. 47(1): 217.

14- Oliveira, D.C. and de Lencastre, H. (2011). Methicillin-resistance in Staphylococcus aureus is not affected by the over expression in trans of the mecA gene 
repressor: a surprising observation. PLoS. 17- Robles, B.F., Nóbrega, D.B., Guimarães, ONE. 6(8): e23287.

F.F., Wanderley, G.G. and Langoni, H. (2014). $\quad \beta$-lactamase detection in

15- Ba, X., Harrison, E.M., Edwards, G.F., Holden, M.T.G., Anders Rhod Larsen, A.R., Petersen, A., Skov, R.L., Sharon J. Peacock, S.J., Parkhill, J., Gavin K. Paterson, G.K. and Mark A. (2014). Novel mutations in penicillin-binding protein genes in clinical Staphylococcus aureus isolates that are methicillin resistant on susceptibility testing but lack the mec gene. J. Antimicrob. Chemother. 69: 594-597.

16- Kaase, M., Lenga, S., Friedrich, S., Szabados, F., Sakinc, T., Kleine, B. and Gatermann, S.G. (2008). Comparison of phenotypic methods for penicillinase detection in Staphylococcus aureus. Clin. Microbiol. Infect. 14: 614-616.

Staphylococcus aureus and coagulasenegative Staphylococcus isolated from bovine mastitis. Pesq. Vet. Bras. 34(4): 325328.

18- Devapriya, F., Ramesh, R., Khan, A.K.S. and Shanmugam, J. (2013). $\beta$-Lactamase production of Staphylococcus aureus: a comparison study of different iodometric methods. Gulf. Med. J. 2: 16-21.

19- Gill, V.J., Manning, C.B. and Ingalls, C.M. (1981). Correlation of penicillin minimum inhibitory concentrations and penicillin zone edge appearance with Staphylococcal $\beta$-lactamase production. J. Clinc. Microbiol. 437-440. 


\section{تشخيص انزيمات البيتا لاكتاميز المنتج من قبل المكورات العنقودية الذهبية المقاومة للمثيسيلين من المين المكن}

الخلاصـــة

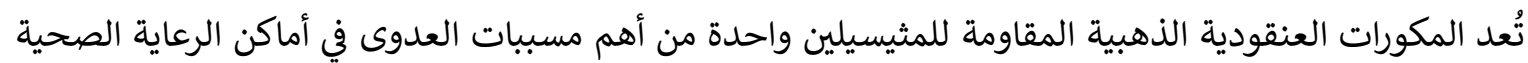

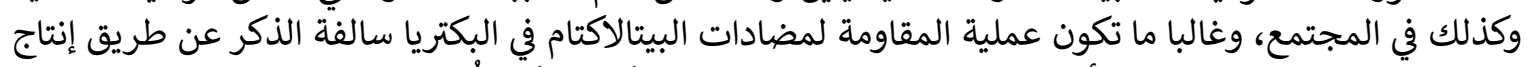

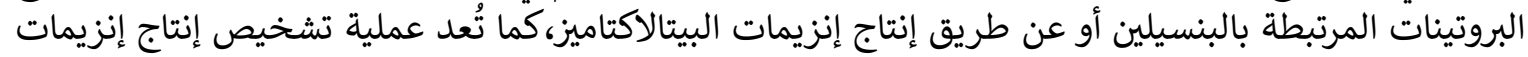

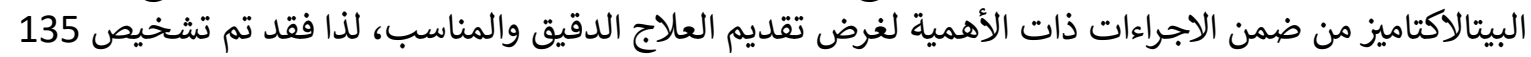

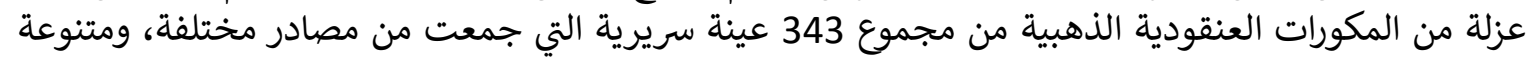

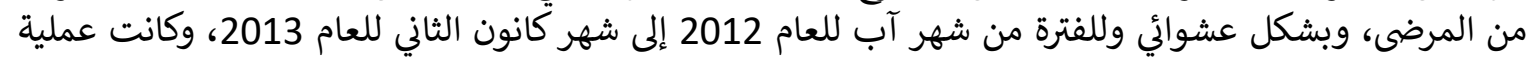

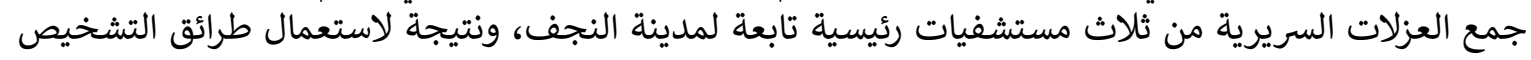

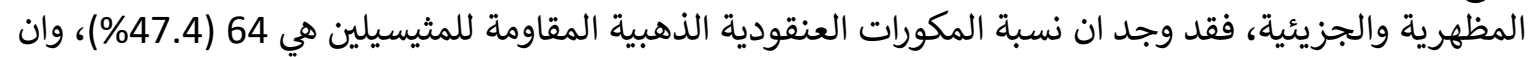

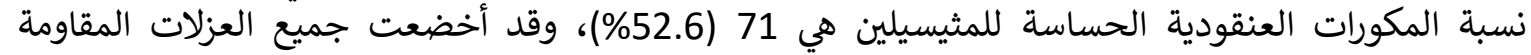

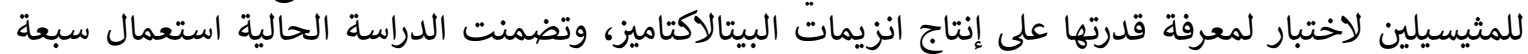

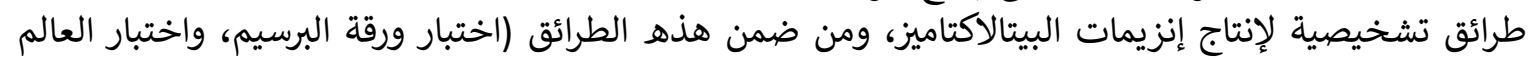

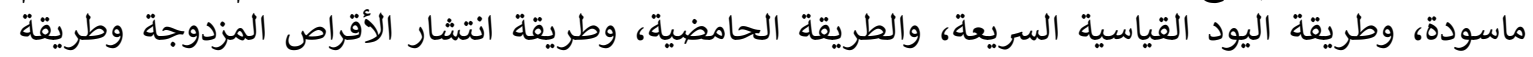

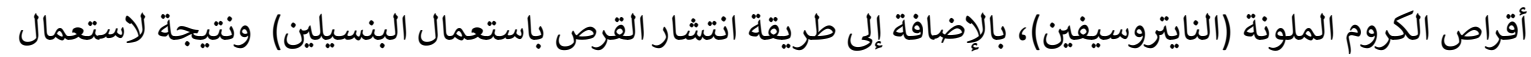

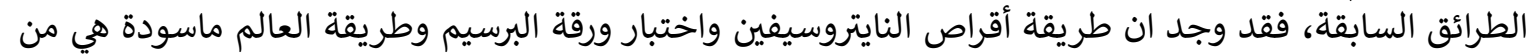

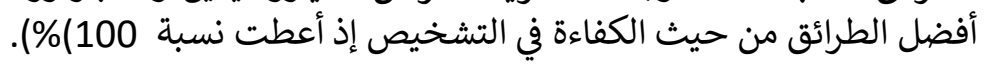

